\title{
Determinación de la Densidad de Entrecruzamiento de Poliuretanos Obtenidos a partir de Aceite de Ricino Modificado por Transesterificación
}

\author{
Manuel F. Valero \\ Universidad de la Sabana, Colombia \\ Jorge E. Pulido, Álvaro Ramírez \\ Universidad Industrial de Santander, Colombia

\section{Zhengdong Cheng \\ Texas A\&M University, US}

\begin{abstract}
Resumen: Se obtuvo una gama de polioles a partir del aceite de ricino modificado por transesterificación con pentaeritriol y glicerina. Los productos resultantes fueron caracterizados físico-químicamente. Se sintetizaron elastómeros de poliuretano a partir de los polioles derivados del aceite de ricino modificado. La densidad de entrecruzamiento se determinó por ensayos de hinchamiento a partir de la ecuación de Flory-Rehner apoyada en la espectroscopia MALDI TOF MS para determinar la estructura química de la unidad repetitiva promedio del poliuretano. Se estudió la variación de la densidad de entrecruzamiento de los poliuretanos con base en el índice de hidroxilo del poliol utilizado en la síntesis. Los resultados muestran que los PU obtenidos a través de la modificación del aceite con pentaeritriol tienen una mayor densidad de entrecruzamiento que los PU obtenidos a partir del aceite original y de los polioles derivados de la reacción de transesterificación del aceite con glicerina, debido a que estos polioles poseen una mayor funcionalidad hidroxílica.
\end{abstract}

Palabras-claves: Aceite de ricino, poliuretanos, densidad de entrecruzamiento.

\section{Determination of Crosslink Density of Polyurethanes Obtained from Castor Oil Modified by Transesterification}

Abstract: Two series of polyols were obtained from castor oil modified by transesterification with various amounts of pentaerythritol and glycerol. The resultant polyol properties were characterized as a function of the hydroxyl functionality. Polyurethane elastomers (PU) were synthesized from the two series of polyols obtained. The crosslink density of those polyurethanes was determined by swelling tests data adjusted to the Flory-Rehner's equation and according to the elasticity theory. The variation of the crosslink density of polyurethanes was studied by following the hydroxyl value of the modified polyol utilized in the synthesis and based on the average structure of each polyurethane repeating unit, as determined by the method of matrix-assisted laser desorption/ionization time-of-flight mass spectroscopy (MALDI TOF MS). The results show that the PU obtained through castor oil modified with pentaerythritol had a higher crosslink density than PU obtained from unmodified castor oil and from PU prepared by transesterification reaction of castor oil with glycerol. PU based on polyols with the higher hydroxyl functionality presented the higher crosslink density.

Keywords: Castor oil modified, polyurethanes, crosslink density.

\section{Introducción}

Numerosos estudios se han realizado empleando el aceite de ricino como materia prima para la obtención de poliuretanos elastoméricos, debido a su estructura química y funcionalidad resultan polímeros entrecruzados cuyas propiedades están controladas por la densidad de entrecruzamiento ${ }^{[1-5]}$. Los poliuretanos obtenidos a partir del aceite presentan propiedades como bajo módulo de elasticidad, baja resistencia a la rasgada y baja resistencia a tratamientos con altas temperaturas. Estas propiedades son atribuidas principalmente a la menor velocidad de curado del aceite como resultado de la baja funcionalidad y reactividad de los grupos funcionales (hidroxilos secundarios) lo que provoca la formación de un poliuretano con estructura irregular con baja densidad de entrec ruzamiento. Estas características han estado soportadas por ensayos de resistencia al ataque químico que indican que estos materiales poseen una baja densidad de entrecruzamiento. Los resultados del análisis térmico dinamo-mecánico (DMTA) sugieren una estructura de red con bajo grado de entrecruzamiento ${ }^{[8-11]}$.

Para modificar estas propiedades, el aceite se ha modificado por transesterificación con polialcoholes con el fin de obtener un producto de mayor funcionalidad. $\mathrm{Al}$ aumentar esta funcionalidad, se modifican las propiedades físicomecánicas y físico-químicas de poliuretanos, debido a que 
se produce un aumento en la densidad de entrecruzamiento del polímero.

Prashantha y colaboradores ${ }^{[16]}$ que sintetizaron redes interpenetradas de polímero (IPN) a partir de aceite de ricino modificado con glicerina y poli(hidroximetilmetacrilato) (PHEMA). Athawale y colaboradores ${ }^{[22]}$ también utilizaron el aceite de ricino modificado con glicerina, pero con el sistema poliuretano/poli-metilmetacrilato. Ambos grupos de autores encontraron que los IPN's sintetizados mostraron mejores propiedades mecánicas y químicas con relación a los materiales obtenidos con el aceite sin modificación alguna. Sin embargo, al utilizar glicerina para la transesterificación, el triglicérido obtenido posee baja estabilidad térmica y es fácilmente descompuesto a altas temperaturas. Esta desventaja se debe a la presencia de átomos de hidrógeno en la posición $\beta$ relativos a los grupos hidroxilo de la glicerina ${ }^{[13]}$. Este problema puede solucionarse utilizando otro polialcohol que no contenga átomos de hidrógeno en la posición $\beta$, por ejemplo pentaeritriol.

En este trabajo se determinaron las propiedades fisicoquímicas, mecánicas, térmicas y la determinación de la densidad de entrecruzamiento de poliuretanos sintetizados a partir de aceite de ricino modificado por transesterificación con pentaeritriol y glicerina para estudiar el efecto de la estructura del poliol sobre la densidad de entrecruzamiento del poliuretano (PU) en función de la concentración de agente modificador utilizado. La densidad de entrecruzamiento fue obtenida a partir de ensayos de hinchamiento con solventes. Con base en los resultados se obtuvieron correlaciones del índice de hidroxilo del poliol y de la densidad de entrecruzamiento del poliuretano contra la concentración de agente modificador utilizado durante la transesterificación del aceite de ricino.

\section{Experimental}

\section{Materiales}

Los materiales usados en este estudio fueron: Aceite de ricino (índice de hidroxilo: $163 \mathrm{mg} \mathrm{KOH} / \mathrm{g}$ e índice de acidez: 2,33 $\mathrm{mg} \mathrm{KOH} / \mathrm{g}$ ) grado USP, distribuido por Laboratorios León, Bucaramanga. Pentaeritriol marca Fisher, distribuido por Produquímica de Colombia S.A., Bogotá. 4,4-metilendifenilisocianato (MDI), marca Aldrich, distribuido por Arquilab Ltda. Acetona marca Aldrich, distribuido por Arquilab Ltda., Bucaramanga. Glicerina grado USP distribuida por Laboratorios León, Bucaramanga.

\section{Métodos}

Síntesis de los polioles: La transesterificación del aceite de ricino usando pentaeritriol se realizó en un balón-reactor de cuatro bocas equipado con termómetro, agitador mecánico, atmósfera inerte y condensador de reflujo. El balónreactor se cargó con el aceite de ricino, pentaeritriol y óxido de plomo $(\mathrm{PbO})$ como catalizador, en relación $0,05 \%$ ( $\mathrm{PbO} / \mathrm{masa}$ de aceite). Para encontrar las condiciones de re- acción se partió de las condiciones reportadas por Prashantha y colaboradores ${ }^{[16]}$ en el aceite de ricino transesterificado con glicerina. Se hicieron varios experimentos de síntesis variando la temperatura y el tiempo de reacción, y se midió el índice de hidroxilo del poliol resultante. A partir de estos resultados experimentales iniciales se fijaron las siguientes condiciones de reacción; $(210 \pm 5){ }^{\circ} \mathrm{C}$, por 2 horas. Se realizó la transesterificación del aceite con pentaeritriol utilizando según las concentraciones que se describen en la Tabla 1. También, se realizó la transesterificación del aceite con glicerina (bajo las mismas condiciones $)^{[15-17]}$, ver Tabla 1 .

Síntesis de los poliuretanos: Se mezclaron el poliol y el MDI fundido, según la composición necesaria; se utilizó para la síntesis de los poliuretanos una relación $\mathrm{NCO} / \mathrm{OH}=0,7$. La mezcla se agitó y el prepolímero obtenido se sometió a vacío y posteriormente se vierte en un molde de acero hermético de placas paralelas. El período de curado constó de dos etapas, la primera a temperatura ambiente y la segunda a $90{ }^{\circ} \mathrm{C}$, ambas durante 4 horas.

\section{Caracterización de los polioles}

1) Índice de hidroxilo e índice de acidez: Se determinó el índice de hidroxilo de los polioles obtenidos según la norma ASTM D1957-86, con el objetivo de evaluar la concentración de grupos hidroxilos en el aceite de ricino después de la reacción de transesterificación. Se pesan $2 \mathrm{~g}$ de muestra (poliol) en un balón redondo de $250 \mathrm{~mL}$. Se agregan $5 \mathrm{~mL}$ de la solución piridina - anhídrido acético. La muestra se calienta en baño maría durante una hora (la muestra debe agitarse cada 5 minutos aprox.); luego se agregan por la boca del condensador $10 \mathrm{~mL}$ de agua destilada, y se calienta por 10 minutos más. Cuando la muestra este fría se le agregan $25 \mathrm{~mL}$ de butanol neutralizado. Finalmente se titula con una solución de $\mathrm{KOH}$ 0,5 N.

El índice de hidroxilo se calculó según la Ecuación 1:

$$
\begin{aligned}
& \text { Valor hidroxilo }=\left(B+\left(\left(S^{*} A\right) / C\right)-V\right) * S^{-1} * \\
& N^{*} 56,1[\mathrm{mg} \mathrm{KOH} / \mathrm{g} \text { muestra }]
\end{aligned}
$$

donde $\mathrm{A}=$ Solución de $\mathrm{KOH}$ requerida para la titulación del valor ácido $(\mathrm{mL}) ; \mathrm{B}=$ Solución de $\mathrm{KOH}$ requerida para la titulación del blanco $(\mathrm{mL}) ; \mathrm{C}=$ Muestra usada para el valor ácido (g); V = Solución de $\mathrm{KOH}$ requerida para la titulación de la muestra acetilada $(\mathrm{mL})$; y $\mathrm{S}=$ Muestra usada para la acetilación (g).

Se determinó el índice de acidez según la norma ASTM D4662-87, con el fin de evaluar la concentración de grupos carboxilo de los polioles obtenidos en la transesterificación. Se pesan 9 a $11 \mathrm{~g}$ de la muestra. Se le adicionan $10 \mathrm{~mL}$ de piridina neutralizada. La muestra se calienta en baño maría durante una hora (la muestra debe agitarse cada 5 minutos aprox.); luego se agregan por la boca del condensador $10 \mathrm{~mL}$ de agua destilada, y se calienta por 10 minutos más. Cuando la muestra este fría, se le agregan $25 \mathrm{~mL}$ de butanol neutrali-

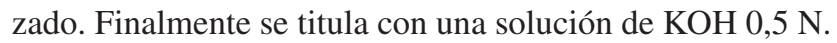


Tabla 1. Índice de hidroxilo, índice de acidez, viscosidad y densidad de los productos obtenidos en función de la concentración de agente modificador (\% en masa).

\begin{tabular}{|c|c|c|c|c|c|}
\hline Nombre del poliol & $\begin{array}{c}\text { Porcentaje en masa de } \\
\text { agente modificador }(\%)\end{array}$ & $\begin{array}{l}\text { Índice de hidroxilo } \\
\text { (mg KOH/g muestra) }\end{array}$ & $\begin{array}{c}\text { Índice de acidez } \\
\text { (mgKOH/g muestra) }\end{array}$ & Viscosidad $^{a}(\mathbf{c p})$ & Densidad $^{\mathrm{b}}\left(\mathrm{g} \cdot \mathrm{mL}^{-1}\right)$ \\
\hline \multicolumn{6}{|l|}{ Pentaeritriol } \\
\hline Penta 0 (P0) & 1,32 & $191 \pm 3$ & $1,05 \pm 0,03$ & $572 \pm 5$ & $0,958 \pm 0,004$ \\
\hline Penta 1 (P1) & 2,64 & $236 \pm 3$ & $0,71 \pm 0,03$ & $732 \pm 5$ & $0,966 \pm 0,004$ \\
\hline Penta 2 (P2) & 5,28 & $250 \pm 3$ & $0,80 \pm 0,03$ & $835 \pm 5$ & $0,971 \pm 0,004$ \\
\hline Penta 3 (P3) & 7,92 & $297 \pm 3$ & $1,22 \pm 0,03$ & $924 \pm 5$ & $0,977 \pm 0,004$ \\
\hline Penta 4 (P4) & 10,56 & $315 \pm 3$ & $1,08 \pm 0,03$ & $992 \pm 5$ & $0,980 \pm 0,004$ \\
\hline \multicolumn{6}{|l|}{ Glicerina } \\
\hline Glice 1 (G1) & 1,75 & $201 \pm 3$ & $0,52 \pm 0,03$ & $660 \pm 5$ & $0,965 \pm 0,004$ \\
\hline Glice 2 (G2) & 3,50 & $219 \pm 3$ & $0,47 \pm 0,03$ & $668 \pm 5$ & $0,965 \pm 0,004$ \\
\hline Glice 3 (G3) & 5,25 & $222 \pm 3$ & $0,38 \pm 0,03$ & $649 \pm 5$ & $0,968 \pm 0,004$ \\
\hline Glice 4 (G4) & 7,00 & $241 \pm 3$ & $0,43 \pm 0,03$ & $636 \pm 5$ & $0,973 \pm 0,004$ \\
\hline Glice 5 (G5) & 9,75 & $250 \pm 3$ & $0,51 \pm 0,03$ & $640 \pm 5$ & $0,980 \pm 0,004$ \\
\hline
\end{tabular}

${ }^{a}$ La viscosidad fue medida a $200 \mathrm{rpm}$, con aguja \#34, cámara $13 \mathrm{R}$ y una temperatura de $20 \pm 3{ }^{\circ} \mathrm{C}$; y ${ }^{\mathrm{b}}$ Para determinar la densidad se utilizó un picnómetro de $1 \mathrm{~mL}$ y posteriormente la muestra se pesó en una balanza digital a una temperatura de $25 \pm 3{ }^{\circ} \mathrm{C}$. (Densidad del agua $1 \mathrm{~g}$. $\mathrm{mL}^{-1}$ ).

2) Viscosidad y densidad: La viscosidad se midió en un viscosímetro marca Brookfield, modelo DV - III + Rheometer, a 200 rpm, utilizando aguja tamaño 31 y la densidad se determinó según la norma ASTM D792-86.

\section{Caracterización de los poliuretanos}

3) Resistencia química: se evaluó según norma ASTM D543-67. Las muestras tomadas fueron circunferencias de $1,6 \mathrm{~cm}$ de diámetro y $0,3 \mathrm{~cm}$ de espesor. Los reactivos usados para tal fin fueron: acetona, agua destilada (a temperatura ambiente y a $70{ }^{\circ} \mathrm{C}$ ), tolueno, amoniaco comercial, ácido clorhídrico al $10 \%$, gasolina y tetracloruro de carbono. Las muestras se sumergieron en cada solvente por 7 días. Durante este tiempo se registraron cambios en la superficie de las muestras. Al final de los 7 días las muestras se secan y se remueve el exceso de solvente, y éstas permanecen durante 24 horas en un desecador. Este procedimiento se llevó a cabo en al menos tres muestras de cada material. El porcentaje de hinchamiento fue calculado usando la Ecuación $2^{[14]]}$ :

$$
\% \text { Hinchamiento }=\left(m_{\mathrm{t}}-m_{\mathrm{i}}\right) * m_{\mathrm{i}}^{-1} * 100
$$

donde $\mathrm{m}_{\mathrm{t}}=$ masa de la muestra en el tiempo $\mathrm{t}$ ( 7 días en este caso) y $\mathrm{m}_{\mathrm{i}}=$ masa de la muestra inicial.

La densidad de entrecruzamiento de algunos poliuretanos sintetizados se determinó por ensayos de hinchamiento. Muestras circulares de dimensiones $(1,6 \pm 0,2) \mathrm{cm}$ de diámetro y $(0,3 \pm 0,1) \mathrm{cm}$ de espesor fueron puestas en acetona (solvente en el cual los materiales presentaron el máximo hinchamiento en las pruebas de resistencia al ataque químico. La acetona posee un parámetro de solubilidad $\delta_{1}=20,7(\mathrm{MPa})^{1 / 2}$ y un volumen molar de $\mathrm{V}_{1}=66,5 \mathrm{~cm}^{3} / \mathrm{mol}$ ) durante 7 días a $27 \pm 2{ }^{\circ} \mathrm{C}$. Después de alcanzar el equilibrio (punto en el cual el grado de hinchamiento no cambia), se remueve el exceso de solvente, y se determinó el cambio de volumen en las muestras.
4) Resistencia a la tracción Uniaxial y dureza Shore A: Las pruebas de resistencia a tensión se realizaron en una máquina universal de ensayos Instron Houns-Field modelo 4204 de carga máxima $50000 \mathrm{~N}$. La velocidad de prueba fue 0,2 in $\min ^{-1}$ a una temperatura $(19 \pm 2){ }^{\circ} \mathrm{C}$, según la norma ASTM D638. Las dimensiones de la muestra fueron de $(40 \times 6 \times 3) \mathrm{mm}^{3}$. Para cada muestra de tres a cinco replicas fueron evaluadas en las mismas condiciones. La dureza Shore A de los materiales se determinó según la norma ASTM D785. Se determinó utilizando un durómetro shore A con punzón, y se tomaron medidas en al menos 10 puntos dentro de la lamina del material.

\section{Resultados y Discusión}

1) Índice de hidroxilo e índice de acidez: el índice de hidroxilo, índice de acidez, viscosidad y densidad de los productos que se utilizaron en la síntesis de poliuretanos se muestran en la Tabla 1. Los valores del índice de hidroxilo producto obtenido cuando el aceite se modificó con glicerina (Glice 3 que contiene una concentración de glicerina de $5,25 \%$ ) coinciden con los reportados por Sherigara y Colaboradores ${ }^{[15]}$. Las propiedades del producto obtenido por Sherigara cuando un equivalente de aceite de ricino reaccionó con un equivalente de glicerina fueron las siguientes: índice de hidroxilo de $223 \mathrm{mg} \mathrm{KOH} / \mathrm{g}$, un índice de acidez de $2,0 \mathrm{mg} \mathrm{KOH} / \mathrm{g}$ y una viscosidad de $486 \mathrm{cP}$ (medida a $30^{\circ} \mathrm{C}$ ). La diferencia en los valores encontrados del índice de acidez y viscosidad se debe posiblemente a las condiciones en las que realizaron los ensayos ${ }^{[15,16]}$.

En la Figura 1 se muestran los valores del índice de hidroxilo para los polioles obtenidos con respecto a la concentración de agente modificador usado en la transesterificación del aceite de ricino. El análisis de los datos experimentales mostró que los resultados se aproximan en gran medida con 


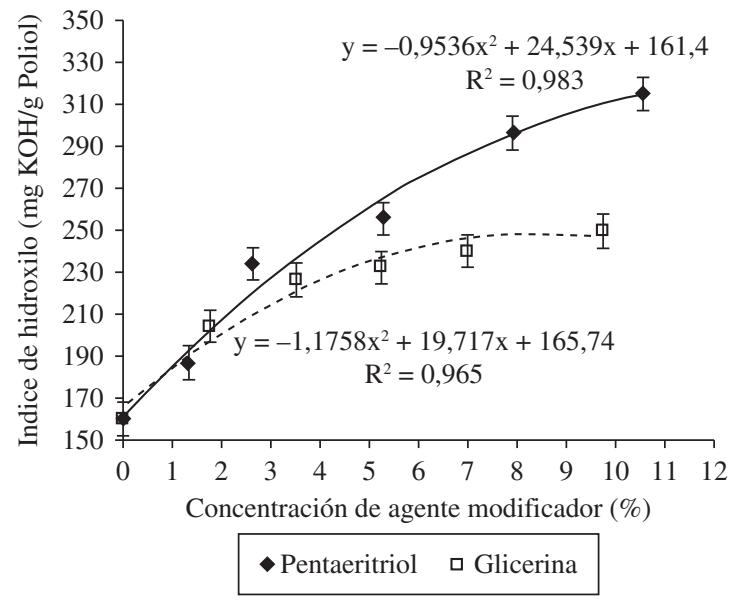

Figura 1. Índice de hidroxilo de los polioles obtenidos a partir de la reacción de transesterificación del aceite de ricino con diferentes concentraciones de agente modificador.

la siguiente Ecuación (3) (parámetros de correlación superiores a 0,965$)$ :

$$
\text { I. } H=a x^{2}+b x+c
$$

donde I.H = es el índice de hidroxilo del poliol, $\mathrm{x}$ es la concentración de agente modificador (\%) y a, b y c son constantes. Las ecuaciones encontradas y los respectivos parámetros de correlación se presentan en la Figura 1.

Se alcanzaron valores más altos del índice de hidroxilo cuando el aceite se modificó con pentaeritriol. Este resultado se debe a que los productos de la reacción de transesterificación del aceite de ricino con glicerina contienen 3 grupos $\mathrm{OH}$; mientras que, los productos de la reacción de transesterificación del aceite con pentaeritriol poseen una funcionalidad de 4 como se observa en la Tabla 2.

2) Viscosidad y densidad: la viscosidad de los polioles obtenidos a través de la modificación del aceite con pentaeritriol aumenta a medida que aumenta la concentración de pentaeritriol. Mientras que, la viscosidad de los polioles obtenidos a través de la modificación del aceite con glicerol aumenta inicialmente, pero luego permanece aproximadamente constante. El incremento en la viscosidad puede ser atribuido al aumento en el peso molecular del producto resultado de la modificación de la estructura del aceite por la reacción de transesterificación. El aumento en la viscosidad también pue-

Tabla 2. Parámetros de interacción de los poliuretanos obtenidos a partir del aceite original y del aceite modificado.

\begin{tabular}{|c|c|c|c|c|c|}
\hline \multicolumn{3}{|c|}{$\mathbf{F}\left((\mathbf{M P a})^{1 / 2 *} \mathrm{~cm}^{3} / \mathrm{mol}\right)$} & \multirow[t]{2}{*}{$\mathrm{V}^{2}\left(\mathrm{~cm}^{3} / \mathbf{m o l}\right)$} & \multirow{2}{*}{$\delta_{2}(\mathbf{M P a})^{1 / 2}$} & \multirow[t]{2}{*}{$\chi_{12}$} \\
\hline Grupo & $\mathbf{F}$ & Número de grupos & & & \\
\hline $\mathrm{CH} 3$ & 420 & 3 & 1157,143 & 19,977 & 0,462 \\
\hline$-\mathrm{CH}_{2-}$ & 280 & 42 & & & \\
\hline$>\mathrm{CH}-$ & 140 & 4 & & & \\
\hline$-\mathrm{CH}=\mathrm{CH}-$ & 444 & 3 & & & \\
\hline -COO- (ester) & 668 & 3 & & & \\
\hline Uretano & 1206 & 2 & & & \\
\hline $\mathrm{OH}$ & 754 & 1 & & & \\
\hline \multirow[t]{2}{*}{ Aromático } & 1517 & 2 & & & \\
\hline & $\sum F i$ & 23116 & & & \\
\hline$-\mathrm{CH}_{3}$ & 420 & 2 & 891,445 & 19,972 & 0,439 \\
\hline$-\mathrm{CH}_{2-}$ & 280 & 29 & & & \\
\hline$>\mathrm{CH}-$ & 140 & 3 & & & \\
\hline$-\mathrm{CH}=\mathrm{CH}-$ & 444 & 2 & & & \\
\hline -COO- (ester) & 668 & 2 & & & \\
\hline Uretano & 1206 & 2 & & & \\
\hline $\mathrm{OH}$ & 754 & 1 & & & \\
\hline \multirow[t]{2}{*}{ Aromático } & 1517 & 2 & & & \\
\hline & $\sum F i$ & 17804 & & & \\
\hline$-\mathrm{CH}_{3}$ & 420 & 3 & 1574,153 & 19,992 & 0,315 \\
\hline$-\mathrm{CH}_{2-}$ & 280 & 47 & & & \\
\hline$>\mathrm{CH}-$ & 140 & 4 & & & \\
\hline$-\mathrm{CH}=\mathrm{CH}-$ & 444 & 3 & & & \\
\hline -COO- (ester) & 668 & 3 & & & \\
\hline Uretano & 1206 & 4 & & & \\
\hline $\mathrm{OH}$ & 754 & 3 & & & \\
\hline \multirow[t]{2}{*}{ Aromático } & 1517 & 4 & & & \\
\hline & $\sum F i$ & 31470 & & & \\
\hline
\end{tabular}


de ser atribuido a la presencia de grupos polares que aumentan las interacciones entre las moléculas.

La densidad de los polioles obtenidos aumento en comparación de la densidad del aceite de ricino sin modificar. La densidad de los polioles obtenidos a partir del aceite modificado con pentaeritriol es mayor que para los polioles obtenidos a partir del aceite modificado con glicerina; este comportamiento obedece a que la densidad del pentaeritriol es mayor que la densidad del glicerol. Los valores reportados de densidad de los polioles se encuentran en un rango entre 0,958 y 0,980 .

3) Resistencia química: los resultados del ensayo de resistencia química de los poliuretanos sintetizados frente a diferentes solventes se determinó que todos los poliuretanos obtenidos muestran resistencia a ácidos y bases (hinchamiento bajo en: ácido clorhídrico al 10\%, ácido acético e hidróxido de sodio al $10 \%$ ). Se comprobó que los poliuretanos no se ven afectados cuando son inmersos en agua bajo el tiempo específico de la prueba. También se pudo determinar que los poliuretanos presentaban el mayor grado de hinchamiento en acetona, atribuible a la polaridad de este solvente.

En la Figura 2 se muestra el grado de hinchamiento de los poliuretanos obtenidos con el aceite de ricino modificado con pentaeritriol frente al solvente evaluado (acetona) ${ }^{[18]}$. El máximo hinchamiento lo presentó el poliuretano obtenido a partir del Penta 0 (PU-P0-0,7), mientras que el mínimo lo registró el poliuretano obtenido a partir del Penta 2 (PU-P2-0,7). Al incrementar la funcionalidad del poliol utilizado en la síntesis del PU disminuye la capacidad de penetración del solvente a la matriz del polímero. Al aumentar la densidad de entrecruzamiento del material, como respuesta al aumento de la funcionalidad del poliol, el material alcanza el equilibrio dinámico a un menor volumen de hinchamiento.

4) Determinación de la densidad de entrecruzamiento: La densidad de entrecruzamiento de los poliuretanos se obtuvo usando la siguiente Ecuación (4) de Flory-Rehner ${ }^{[19]}$ :

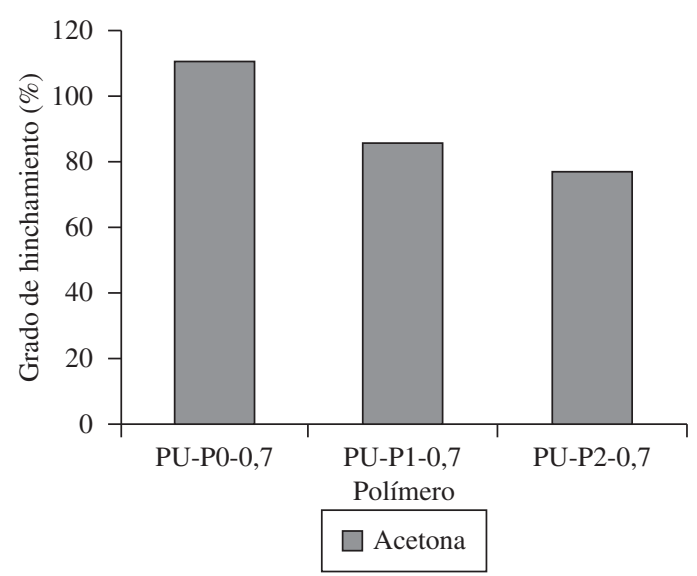

Figura 2. Grado de hinchamiento de los poliuretanos sintetizados a partir del aceite de ricino modificado por transesterificación con pentaeritriol. (La segunda sigla en la nomenclatura indica el poliol que fue utilizado en la síntesis y la tercera sigla indica la relación $\mathrm{NCO} / \mathrm{OH}$ del poliuretano).

$$
\begin{aligned}
& \frac{v_{c}}{V_{o}}\left(m o l / \mathrm{cm}^{3}\right)=-2 *\left[v+\chi_{12} * v^{2}+\right. \\
& \ln (1-v)] *\left[V_{1} *\left(2 * v^{1 / 3}-v\right)\right]^{-1}
\end{aligned}
$$

donde $v_{c}=$ número efectivo de cadenas entrecruzadas; $\mathrm{V}_{1}=$ Volumen molar del solvente; $\chi_{12}=$ parámetro de interacción Solvente-polímero, $v=\frac{\mathrm{V}_{\mathrm{o}}}{\mathrm{V}} ; \mathrm{V}_{\mathrm{o}}=$ Volumen del polímero sin hinchar (seco); y V = Volumen del polímero hinchado en el punto de equilibrio.

El parámetro de interacción $\chi_{12}$ se estimó según la Ecuación 5:

$$
\chi_{12}=V^{1} *(R * T)^{-1} *\left(\delta_{1}-\delta_{2}\right)^{2}
$$

donde $\delta_{1}=$ Parámetro de solubilidad del solvente a una temperatura absoluta de $298 \mathrm{~K}$; y $\delta_{2}=$ Parámetro de solubilidad del polímero a una temperatura absoluta de $298 \mathrm{~K}$. El parámetro de solubilidad del polímero, $\delta_{2}$, fue calculado por el método de contribución de grupos desarrollado por Van Krevelen ${ }^{[20]}$; el método requiere el valor de una constante de atracción molar, $\mathrm{F}_{\mathrm{i}}$, para cada grupo químico en la unidad repetitiva del polímero. Los valores de $\mathrm{F}_{\mathrm{i}}$ fueron tomados de los datos reportados por Hoy y colaboradores ${ }^{[2]}$. $\mathrm{V}_{\mathrm{i}}\left(\mathrm{cm}^{3} / \mathrm{mol}\right)=$ Volumen molar de la unidad repetitiva $\mathrm{y}$ $\mathrm{F}_{\mathrm{i}}\left((\mathrm{MPa})^{1 / 2} *\left(\mathrm{~cm}^{3} / \mathrm{mol}\right)=\right.$ Constantes de atracción molar (Ecuación 6).

$$
\delta_{i}=\Sigma F_{i} * V_{i}^{-1}
$$

En la Tabla 2 se muestran los parámetros de interacción $\chi_{12}$ de los PU, sintetizados a partir del aceite sin modificar y del aceite modificado por transesterificación con glicerina y con pentaeritriol, cuyos valores fueron evaluados usando las Ecuaciones 4 y 5 a partir de las unidades repetitivas de cada poliuretano. Las estructuras de las unidades repetitivas promedio mostradas en la Figura 3 de los poliuretanos obtenidos a partir del aceite original y el aceite modificado se plantearon con base en los resultados de la espectroscopia de masas MALDI TOF.

Los valores de $\chi_{12}, V_{o}, V y \frac{\mathrm{v}_{c}}{V_{o}}$ para los poliuretanos obtenidos a partir del aceite de ricino modificado por transesterificación con glicerina y pentaeritriol se presentan en la Tabla 3. Los valores de la densidad de entrecruzamiento decrecen desde $305 \mathrm{~mol} / \mathrm{m}^{3}$ en el PU-P2-0,7 hasta $179 \mathrm{~mol} / \mathrm{m}^{3}$ para el PU-CO-0,9. Se observa que la densidad de entrecruzamiento aumenta al aumentar la cantidad de agente modificador utilizado para la transesterificación.

La Figura 4 muestra la relación existente entre la densidad de entrecruzamiento y concentración del agente utilizado para la transesterificación del aceite de ricino. Los resultados indican que la densidad de entrecruzamiento es mayor para los poliuretanos sintetizados a partir del aceite de ricino modificado por transesterificación con pentaeritriol. Debido a que estos polioles poseen una mayor funcionalidad hidroxí- 
Del aceite de ricino (PU-CO)

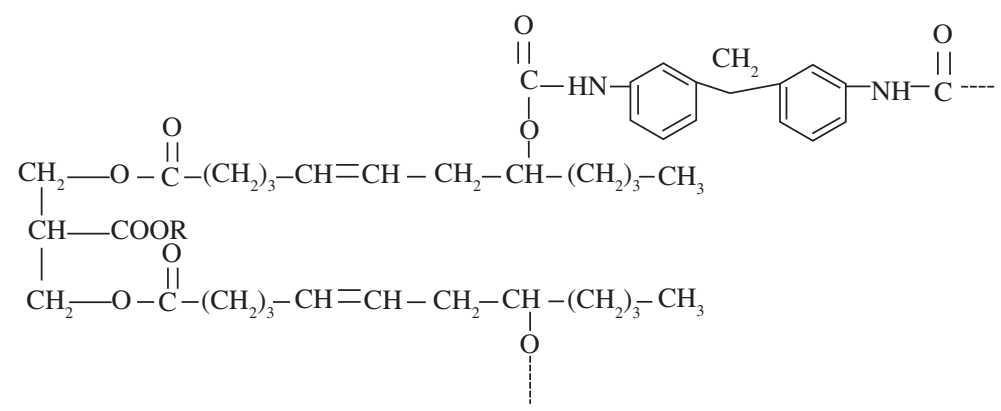

(a)

Del aceite de ricino modificado por transesterificación con glicerina (PU-G)

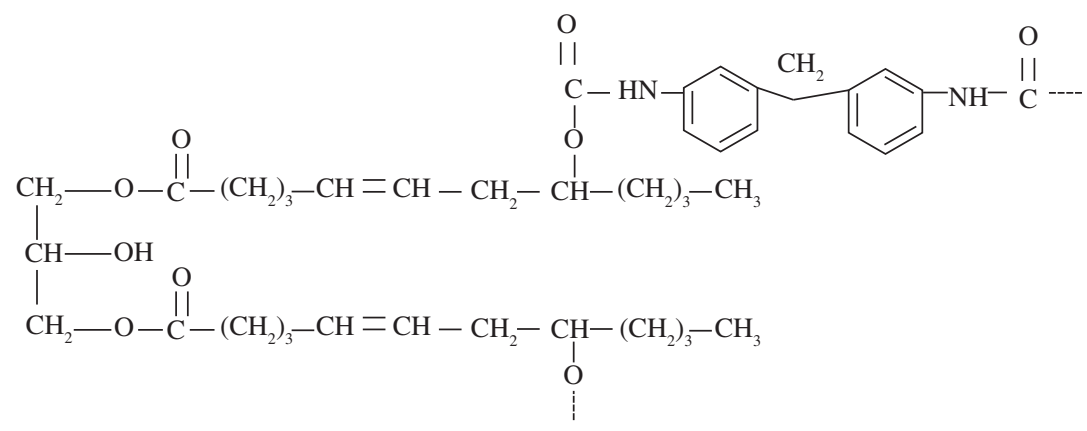

(b)

Del aceite de ricino modificado por transesterificación con pentaeritriol (PU-P)

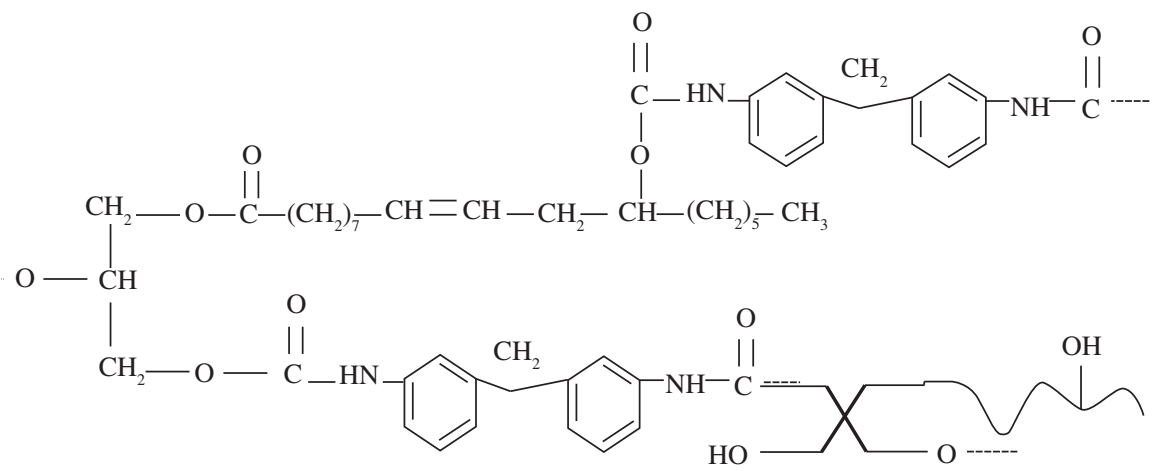

(c)

Figura 3. Unidades repetitivas promedio de los poliuretanos obtenidos a partir de: a) Del aceite de ricino (PU-CO); b) del aceite de ricino modificado por transesterificación con glicerina (PU-G); y c) del aceite de ricino modificado por transesterificación con pentaeritriol (PU-P).

lica, por lo que la posibilidad para formar grupos uretano aumenta.

4) Resistencia a la tracción Uniaxial y dureza Shore A: Los datos obtenidos de tensión de ruptura, elongación de ruptura, módulo de Young y dureza de los poliuretanos obtenidos del aceite de ricino sin modificar y el modificado por transesterificación con pentaeritritol y glicerina se reportan en la Tabla 4. Se observa un incremento en el módulo de elasticidad, la tensión de ruptura y la dureza Shore A, a medida que se utiliza - para la síntesis del material- un poliol de mayor índice de hidroxilo. El incremento en los valores de la tensión de ruptura y módulo de Young fue: de 1960 y $226 \mathrm{KPa}$ para el poliuretano obtenido del aceite de ricino sin modi- ficar, hasta 7323 y $1120 \mathrm{KPa}$ para el poliuretano obtenido del poliol 2, respectivamente. Éste comportamiento se debe a que al aumentar la cantidad de grupos hidroxilo del aceite de ricino por transesterificación aumenta la rigidez del material debido al incremento en la densidad de entrecruzamiento del poliuretano. $\mathrm{Al}$ aumentar la funcionalidad del poliol también se incrementan las interacciones entre los segmentos del poliuretano.

Las curvas de tensión-deformación de los poliuretanos obtenidos muestran características típicas de materiales tipo elastómeros: bajo módulo de Young, elongación de ruptura elevada y valores medios del esfuerzo de ruptura. Los resultados se muestran en la Figura 5. La energía de ruptura de los 
materiales (tenacidad) - obtenida del área bajo la curva de la correspondiente curva de tensión-deformación - varía de la siguiente manera para los poliuretanos obtenidos, de mayor a menor: PU obtenido por transesterificación del aceite con pentaeritritol $>$ PU obtenido por transesterificación del aceite con glicerol > PU obtenido del aceite de ricino sin modificar.

Tabla 3. Dependencia de la densidad de entrecruzamiento $\left(\frac{v_{c}}{V_{o}}\left(m o l / \mathrm{m}^{3}\right)\right)$ de los poliuretanos obtenidos del aceite modificado por transesterificación con glicerina y pentaeritriol.

\begin{tabular}{|c|c|c|c|c|}
\hline Poliuretano & $\chi_{12}^{a}$ & $\mathbf{V}_{\mathbf{o}}\left(\mathbf{c m}^{3}\right)^{\mathbf{b}}$ & $\begin{array}{c}\mathrm{V} \\
\left(\mathrm{cm}^{3}\right)^{\mathrm{c}}\end{array}$ & $\frac{\mathbf{v}^{c}}{V_{0}}\left(\mathrm{~mol} / \mathrm{m}^{3}\right)^{d}$ \\
\hline PU-CO- $0,9^{\mathrm{e}}$ & 0,462 & 0,603 & 1,272 & 179,19 \\
\hline PU-G1-0,7f & 0,439 & 0,567 & 1,196 & 193,38 \\
\hline PU-G2-0, $7^{\mathrm{f}}$ & 0,439 & 0,615 & 1,259 & 213,67 \\
\hline PU-G3-0, $7^{\mathrm{f}}$ & 0,439 & 0,651 & 1,292 & 237,21 \\
\hline PU-P0-0, $7^{\mathrm{f}}$ & 0,315 & 0,663 & 1,484 & 229,63 \\
\hline PU-P1-0, $7^{\mathrm{f}}$ & 0,315 & 0,640 & 1,362 & 263,30 \\
\hline PU-P2-0, $7^{\mathrm{f}}$ & 0,315 & 0,573 & 1,160 & 305,15 \\
\hline
\end{tabular}

aParámetro de interacción; ${ }^{b}$ volumen del polímero sin hinchar (seco); ${ }^{\mathrm{c}}$ volumen del polímero hinchado en el punto de equilibrio; dnúmero efectivo de cadenas entrecruzadas (densidad de entrecruzamiento); ${ }^{\mathrm{e}}$ relación $\mathrm{NCO} / \mathrm{OH}=0,7 ; \mathrm{y}{ }^{\mathrm{f}}$ relación $\mathrm{NCO} / \mathrm{OH}=0,9$.

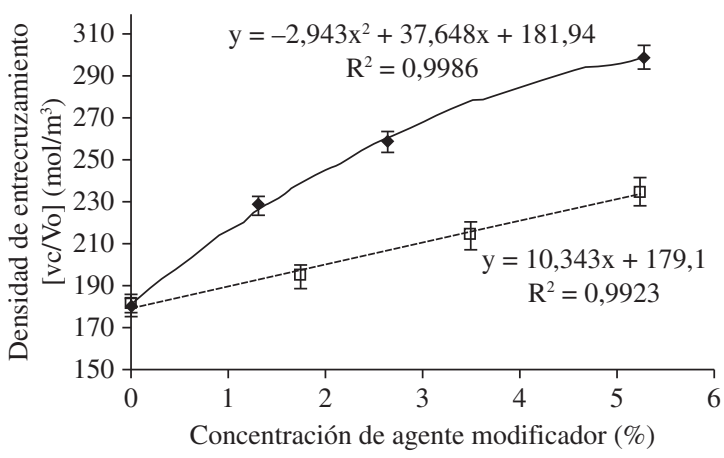

$$
\begin{aligned}
& \text { Poliuretano obtenido a partir del aceite de ricino } \\
& \text { modificado por transesterificación con pentaeritriol } \\
& \text { Poliuretano obtenido a partir del aceite de ricino } \\
& \text { modificado por transesterificación con glicerina }
\end{aligned}
$$

Figura 4. Relación entre la densidad de entrecruzamiento y la concentración de agente modificador (pentaeritriol o glicerina) para los poliuretanos sintetizados a partir de aceite modificado por transesterificación (relación $\mathrm{NCO} / \mathrm{OH}=0,7)$.

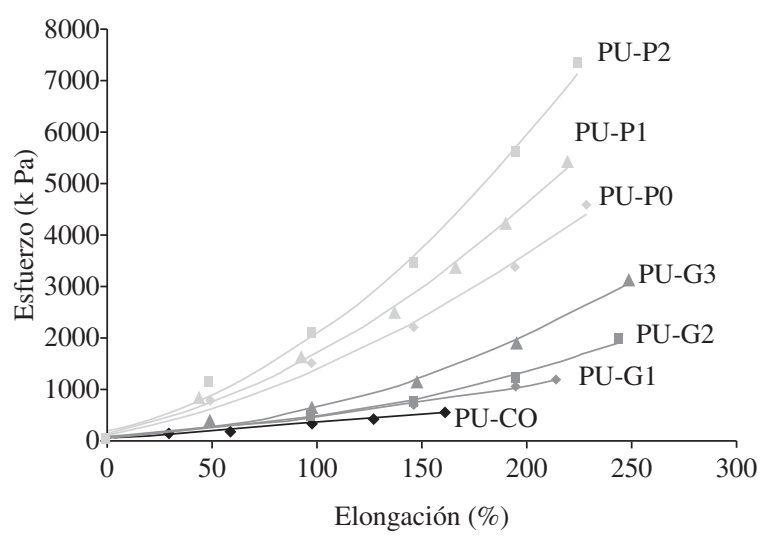

Figura 5. Curvas tensión-deformación de poliuretanos a partir del aceite de ricino sin modificar, poliol 0 y poliol 1 . Relación $\mathrm{NCO} / \mathrm{OH}=0,7$ (excepto el poliuretano del aceite de ricino que se sintetizó con una relación molar $\mathrm{NCO} / \mathrm{OH}=0,9)$.

Un incremento en la energía de ruptura indica que el material tiene una mejor capacidad de absorber la energía.

\section{Conclusiones}

Se determinó la densidad de entrecruzamiento de poliuretanos sintetizados a partir del aceite de ricino original y el aceite de ricino modificado por transesterificación con pentaeritriol y glicerina, a partir de ensayos de entrecruzamiento. Los resultados muestran que la densidad de entrecruzamiento de los poliuretanos obtenidos a partir del aceite de ricino modificado con pentaeritriol se incrementa al aumentar la concentración de pentaeritriol, debido a que aumenta el índice de hidroxilo del poliol resultante. Al aumentar la funcionalidad aumenta la probabilidad de que los grupos uretano del diisocianato reaccionen con los grupos hidroxilo del poliol, lo que promueve la formación de una red de poliuretano que posee una mayor densidad de entrecruzamiento.

\section{Agradecimientos}

Este trabajo fue financiado por Colciencias-SENA-UIS (Proyecto 1102-08-1). Los autores agradecen al profesor Zhengdong Cheng de la Universidad Texas A\&M por la ayuda en la realización de ensayos y pruebas de caracterización.

Tabla 4. Propiedades mecánicas de los poliuretanos obtenidos a partir del aceite de ricino modificado por transesterificación.

\begin{tabular}{ccccc}
\hline Poliuretano & $\sigma(\mathbf{K P a})^{\mathbf{a}}$ & $\epsilon(\%)^{\mathbf{b}}$ & ${\mathbf{E ~}(\mathbf{K P a})^{\mathbf{c}}}$ & Dureza Shore A $^{\mathbf{c}}$ \\
\hline PU-P2-0,7 & $7323 \pm 4,9$ & $224,6 \pm 8,4$ & $1120 \pm 6,9$ & $64 \pm 2$ \\
PU-P1-0,7 & $5428 \pm 3,6$ & $219,7 \pm 7,7$ & $818 \pm 5,6$ & $60 \pm 2$ \\
PU-P0-0,7 & $4573 \pm 3,1$ & $229,5 \pm 6,8$ & $754 \pm 5,1$ & $50 \pm 2$ \\
PU-G3-0,7 & $3141 \pm 3,6$ & $249,0 \pm 6,5$ & $377 \pm 4,6$ & $50 \pm 2$ \\
PU-G2-0,7 & $1960 \pm 3,0$ & $244,1 \pm 7,3$ & $226 \pm 4,0$ & $48 \pm 2$ \\
PU-G1-0,7 & $1163 \pm 3,1$ & $214,8 \pm 6,5$ & $258 \pm 4,1$ & $38 \pm 2$ \\
PU-CO-0,9 & $553 \pm 3,1$ & $161,1 \pm 9,2$ & $201 \pm 5,1$ & $38 \pm 2$ \\
\hline
\end{tabular}

aTensión de ruptura; ${ }^{\text {} e l o n g a c i o ́ n ~ d e ~ r u p t u r a ; ~ ' ~} \mathrm{c}$ ódulo de Young; ${ }^{\mathrm{e}}$ relación $\mathrm{NCO} / \mathrm{OH}=0,7$; y ${ }^{\mathrm{f}}$ relación $\mathrm{NCO} / \mathrm{OH}=0,9$. 


\section{Bibliografía}

1. Kumar, V. G.; Rao, M. R.; Guruprasad, T. R. \& Rao, K. V. C. - J. Appl. Polym. Sci., 34, p.1803 (1987).

2. Patel, P. \& Suthar, B. - Polymer Journal, 23, p.399 (1987).

3. Patel, P. \& Suthar, B. - Polymer, 31 (1990).

4. Suthar, B. - Termochimica Acta, 228, p.213-218, (1993).

5. Pandit, S. B.; Kulkarni, S. S. \& Nadkarni, V. M. - Macromolecules, 27 (16), p.4595 (1994).

6. Trân, N. B.; Vialle, J. \& Pham, Q. T. - Polymer, 38, (10), p.2467 (1997).

7. Bai, S, Khakhar, S. V. \& Nadkarni, V. M. - Polymer, 38 (17), p.4319 (1997).

8. Sperling, L. H. \& Mishra, V. Polymers for Advanced Technologies, 7, (4), p.197-208, (1998).

9. Siddaramaiah, J. - J. Appl. Polym. Sci. (68), p.1739 (1998).

10. Siddaramaiah, J. - Polymer, (63), p.305 (1999).

11. Singh, P.; Kaushik, A. \& Gupta, P. - Journal of Reinforced Plastics and Composites. 24 (14), (2005).

12. Somani, K.; Kansara, S.; Parmar, R. \& Patel, N. - International Journal of Polymeric Materials. (53), p.283 (2004).
13. Yeganeh, H. \& Mehdizadeh M. R. - European Polymer Journal. (40), p.1233 (2004).

14. Bhabhe, M. D. \& Athawale, V. D. - J.Appl.Polym. Sci. (69), p.1451 (1998).

15. Sanmathi, C. S.; Prasannakumar, S. \& Sherigara, B. S. - J. Appl. Polym. Sci. 94, p.1029-1034, (2004).

16. Prashantha, P. K.; Pai, V. K.; Sherigara, B. S. \& Prasannakumar, S. - Bull. Mater. Sci. 24, (5), p.535-538 (2001).

17. Somani, K. P.; Kansara, S. S.; Patel, N. K. \& Rakshit, A. K. - International Journal of Adhesion. 23, p.269-275, (2003).

18. Yongshang, L. U.; Lina, Z.; Xufeng, Z. \& Yan, Z. - Polymer. 44, p.6689 (2003).

19. Flory, P. J. - J.Chem. Phys. 18, p.108 (1950)

20. van Krevelen, D. W. - Properties of Polymers, 3rd edition, Elsevier, Amsterdam, (1990).

21. Hoy, K. L. - J. Paint Technol., 42, p.76 (1970)

22. Athawale, V. \& Kolekar, S. - European polymer journal. 34, (10), p.1447-1451 (1998).

Enviado: $23 / 08 / 07$

Reenviado: 26/08/08

Aceito: 01/09/08 